\title{
Cognitive slowing in Parkinson's disease resolves after practice
}

\author{
D Z Press, D J Mechanic, D Tarsy, D S Manoach
}

J Neurol Neurosurg Psychiatry 2002;73:524-528

See end of article for authors' affiliations

Correspondence to

Dr D Z Press, Beth Israel

Deaconess Medical Center,

330 Brookline Ave,

KS-227, Boston, MA

02215, USA

dpress@

caregroup.harvard.edu

Received

17 January 2002

In revised form 15 July

2002

Accepted 16 July 2002
Objective: To assess the effect of dopaminergic repletion on working memory in Parkinson's disease. Methods: The role of dopaminergic state on working memory in patients with Parkinson's disease was determined using the Sternberg item recognition paradigm, a continuous performance task that dissociates the motor and cognitive components of response time. Ten patients with Parkinson's disease were tested in an "on" state (on dopaminergic drug treatment) and a practical "off" state in two sessions held one week apart in counterbalanced order; 10 controls matched for age and education were studied at the same time points.

Results: Patients with Parkinson's disease showed impaired working memory, independent of motor slowing. During session 1, the performance of the patients was worse than the controls, regardless of dopaminergic state. The patients showed a significant improvement in the cognitive component of task performance during the second session, such that they no longer differed from the controls. The performance of the control subjects remained stable over the two sessions.

Conclusions: Working memory performance of patients with Parkinson's disease did not change in association with dopaminergic state; rather, the performance improved over time. The pattern of improvement over time suggests a delay in proceduralising the task, similar to the deficits shown by such patients in procedural learning of other tasks.
W hile Parkinson's disease primarily impairs motor function, numerous studies have shown that it also affects cognition. Potential causes of cognitive deficit include incipient dementia, ${ }^{1}$ depression, ${ }^{2}$ and the adverse effects of drugs. ${ }^{3}$ However, even untreated, non-depressed, non-demented patients can show cognitive deficits. ${ }^{45}$ Domains of cognition that are related to frontal network function appear to be most affected, including executive function, ${ }^{6}$ set switching, ${ }^{7}$ and working memory. ${ }^{8-11}$ Working memory refers to the ability to maintain information "on-line" and manipulate it in the service of guiding behaviour. ${ }^{12}$ Working memory is thought to be subserved by components of a frontostriatal circuit that includes the dorsolateral prefrontal cortex (DLPFC) and the caudate nucleus. ${ }^{13}$ Dopaminergic projections to the DLPFC, ${ }^{14}$ and possibly also to the caudate nucleus, ${ }^{15}$ play a modulatory role in the function of the working memory network. In this framework, working memory deficits in Parkinson's disease could result from altered dopaminergic innervation of either the DLPFC, though the mesocortical pathway, or the caudate nucleus, through the nigrostriatal pathway. Recent findings that dopamine levels in DLPFC are actually increased in early untreated Parkinson's disease ${ }^{16}$ cast doubt on a cortical dopaminergic deficiency, but either a deficit of dopamine in the caudate nucleus or deficiencies of other neurotransmitters projecting to the DLPFC ${ }^{17-19}$ are possible mechanisms for working memory deficits.

Dopaminergic repletion improves the motor symptoms of Parkinson's disease, but its effects on working memory are unclear. Gotham et al studied patients with Parkinson's disease using various tasks that require working memory, including the Wisconsin card sorting test and the controlled oral word association test. ${ }^{8}$ The patients performed worse than control subjects but their performance was not different in "on" (that is, on dopaminergic drug treatment) versus "off" states. In contrast, Lange and colleagues ${ }^{20}$ showed that dopaminergic repletion improved both set switching and working memory performance on the Tower of London task compared with levodopa withdrawal in advanced Parkinson's disease.
The variability of performance in working memory tasks in patients with Parkinson's disease might reflect several factors including differing task demands and patient characteristics. In addition to working memory, the tasks employed in these studies also require the use of other cognitive functions such as spatial planning and mental imagery, so it is not possible to attribute the findings definitively to working memory.

Several studies have employed the Sternberg item recognition paradigm $(\text { SIRP) })^{21}$ to measure motor and working memory function independently in patients with Parkinson's disease. ${ }^{22-24}$ The SIRP is a choice-reaction time test that dissociates the motor and cognitive components of response time. Accurate responses are predicated upon a temporarily stored representation of the targets that must be maintained in working memory for the duration of the trial. Sternberg has shown that there is a linear relation between response time and the number of targets the subject must keep "on-line" ${ }^{21}$ The slope of the linear function provides a measure of the cognitive component of response time (the increase in response time with each increment in working memory load), and the $y$ intercept provides a measure of the motor component of response time. The SIRP reliably activates the DLPFC in fMRI studies of normal subjects ${ }^{25}{ }^{26}$ and is repeatable with minimal practice effects. ${ }^{27}$

Previous studies of SIRP in Parkinson's disease have yielded discrepant findings. Poewe and colleagues showed that patients with Parkinson's disease in the "off" state had motor slowing but intact working memory, while in the "on" state their motor slowing resolved but their working memory worsened. ${ }^{22}$ This study may have been confounded by an order effect, as the Parkinson's disease patients were always tested first in the "off" state and then in the "on" state. Rafal and

Abbreviations: DLPFC, dorsolateral prefrontal cortex; SIRP, Sternberg item recognition paradigm; UPDRS, unified Parkinson's disease rating scale 
Table 1 Demographic data on the patients and controls

\begin{tabular}{lll}
\hline Variable & PD subjects $(n=10)$ & Controls $(n=10)$ \\
\hline Age (years), with range & $63(5.7), 54$ to 73 & $61.6(7.7), 50$ to 73 \\
Years of education & $15.6(4.2)$ & $15.9(2.7)$ \\
Sex (M:F) & $8: 2$ & $5: 5$ \\
Years with PD & $8.0(6.2)$ & $\mathrm{NA}$ \\
Estimated verbal IQ & $127.7(5.0)$ & $127.8(5.2)$ \\
BDI & $4.8(1.4)$ & $2.4(2.2)$ \\
\hline
\end{tabular}

Values are mean $(S D)$ or $n$.

$B D I$, Beck depression inventory; IQ, intelligence quotient; PD,

Parkinson's disease.

colleagues employed the SIRP in rapidly fluctuating Parkinson's disease and found motor slowing without cognitive slowing in the "off" relative to the "on" state. ${ }^{23}$ This study lacked an age matched control group and did not screen for dementia.

In the current study we investigated the role of dopaminergic repletion on working memory performance using the SIRP. We addressed the potential confounding variables by testing patients with Parkinson's disease in a practical "off" state (after a 12 hour period of drug withdrawal) and an "on" state (one hour after their drug treatment was given) in a counterbalanced order, one week apart. Age matched controls were tested at the same time points. All subjects were screened for dementia and depression. We hypothesised that subjects with Parkinson's disease would have impaired working memory in the "off" state and that these deficits would improve with dopaminergic repletion.

\section{METHODS}

\section{Subjects}

The protocol was approved by the local committee for clinical investigations, and informed consent was obtained from all subjects. Ten patients with Parkinson's disease were recruited from the movement disorders clinic at Beth Israel Deaconess Medical Center by advertisement. All patients met the NINDS diagnostic criteria for probable Parkinson's disease ${ }^{28}$ and had shown a good clinical response to treatment with levodopa. Patients had mild to moderate bilateral disease, stage II-III in the Hoehn and Yahr scale. ${ }^{29}$ At enrolment, nine were receiving levodopa/carbidopa, three were receiving dopamine agonists (one as monotherapy), three were receiving anticholinergic drugs (discontinued for at least 24 hours before testing), one was receiving amantadine, and one was receiving tolcapone.

Ten control subjects (four spouses and six members of the hospital community) were also recruited.

All subjects were screened for depression with the Beck depression inventory ${ }^{30}$ and excluded if the score suggested a mood disorder $(\geqslant 10)$. To screen for dementia, all subjects performed the Mattis dementia rating scale $^{31}$ and were excluded if they showed any evidence of impairment (total score $\leqslant 135$ ). Premorbid intelligence quotient was estimated using the American new adult reading test (ANART). ${ }^{32}$ The demographic and screening data for all subjects are presented in table 1 . There were no significant differences between Parkinson's disease patients and controls in age, estimated IQ, or years of education.

\section{Sternberg item recognition paradigm}

The SIRP was presented using MacStim ${ }^{\mathrm{TM}}$ (Victoria, Australia) on a Macintosh PowerBook (Apple computers Inc, Cupertino, California, USA), and responses were indicated by button presses on a response box. Subjects were visually presented with the digits to be remembered (the memory set), with memory set sizes of one, three, or five digits. They were then presented with trials consisting of the presentation of a single digit and were asked to indicate by pressing a key whether each trial digit was a member of the memorised set (a target) or not (a foil).

A block consisted of the presentation of the memory set followed by 20 trials, half consisting of targets and half of foils, in random order (the fixed set form of the SIRP). In order to respond correctly, subjects had to maintain the memorised set "on-line" for the duration of the block. Each testing session began with practice consisting of one block of each set size. The subjects then performed two blocks of each set size (one, three, and five) in random order for a total of six blocks or 120 trials. The dependent variables were accuracy and response times from correct trials.

\section{Procedure}

For the practical "off" state, Parkinson's disease patients were tested in the morning after an overnight ( 12 hour) period without drug treatment. For the "on" state, they took only levodopa/carbidopa one hour before testing, at a dose clinically equivalent to their usual morning medical regimen (selected by one author, DT, blinded to the test order). The motor score from the unified Parkinson's disease rating scale (UPDRS) was measured at the start of each session. The order of testing was counterbalanced, with five patients tested in the "off" state first and five in the "on" state first. The two test sessions were separated by one week. Control subjects were also tested during two sessions, one week apart.

\section{Statistical analysis}

Response time data were analysed by a randomised block analysis of variance, using the JMP statistical program (version 3.0, SAS Institute, Cary, North Carolina, USA). Set size was a continuous variable; group and session were factors; subjects nested within group was the random factor. Drug state ("on" $v$ "off") was examined in the Parkinson's disease subjects. In these analyses, the set size term represents the slope, or the cognitive, working memory component of the response time and was considered to be a continuous variable. The y intercept term represents the motor component of the response time. A smaller slope indicates a smaller increment in response time for each additional item held in the working memory, and more efficient cognitive performance. A smaller $y$ intercept indicates a faster motor component of the response time.

Response accuracy was compared using repeated measures analyses of variance with drug state ("on" $v$ "off") or session (first $v$ second) and set size (one, three, and five items) as repeated measures and group (normal $v$ Parkinson's disease) as the between factor.

\section{RESULTS}

\section{Motor symptoms in the Parkinson group}

Patients with Parkinson's disease had significantly higher UPDRS motor scores in the "off" state than in the "on" state (UPDRS “on", 12.3; UPDRS “off", 22.0; mean difference -9.7; $\mathrm{t}(9)=5.72, \mathrm{p}<0.001)$, indicating that the drug withdrawal did increase motor impairment.

\section{Reaction time}

Contrary to our initial hypothesis, in the Parkinson's disease group there was no effect of dopaminergic state on either the working memory component (drug state $\times$ set size effect, $\mathrm{F}(\mathrm{l}, 9)=0.09, \mathrm{p}=0.77:$ "on", $\mu$ (mean $(\mathrm{SD})=0.067$ (0.016); "off", $\mu=0.063(0.016)$ ) or the motor component (drug state effect $\mathrm{F}(1,9)=0.02, \mathrm{p}=0.89$ : “on", $\mu=0.616(0.055)$; “off", $\mu=0.622(0.055))$ of the response time. Comparing Parkinson's disease patients and controls, there was no significant difference between groups in the motor component (group main effect: $F(1,18)=1.39, p=0.25)$ but there was a trend toward a slower working memory component (group $\times$ set size 

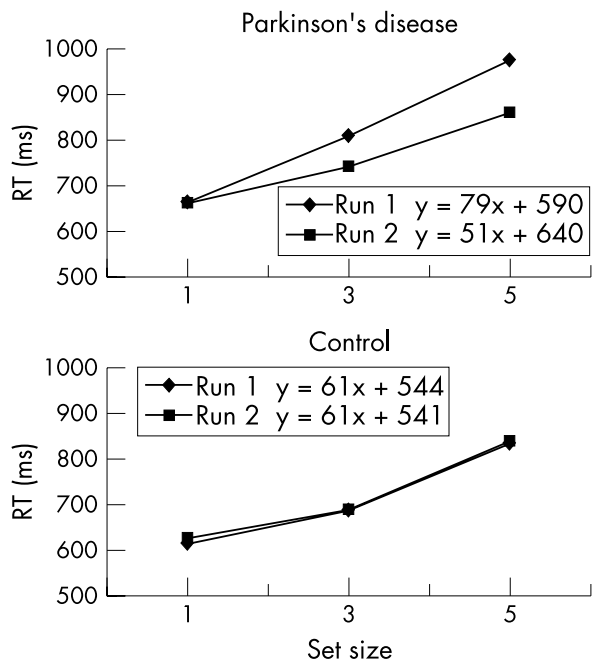

Figure 1 Results from the Sternberg item recognition paradigm (SIRP) showing the increase in response time (RT) with increasing working memory load (set size). Parkinson's disease patients had a significant improvement in their working memory at run 2 , as demonstrated by a decrease in slope. The regression equations are shown in terms of $y=m x+b$ with $m$ representing the slope (ms/item) and $b$ representing the $y$ intercept (ms).

interaction: $F(1,18)=2.6, p=0.10)$. This trend was accounted for by the first session only. There was a dramatic difference in the two groups' working memory performances across sessions (group $\times$ set size $\times$ run interaction: $\mathrm{F}(1,18)=15.2, \mathrm{p}<0.0001)$. While the Parkinson's disease group had a significantly higher slope (less efficient working memory) than the controls at the first session (group $\times$ set size interaction: $F(1,18)=12.9, p=0.0003)$, this difference resolved completely by the second session, in which the Parkinson's disease group showed a trend to a lower slope (group $\times$ set size interaction: $\mathrm{F}(\mathrm{l}, 18)=2.8, \mathrm{p}=0.09$ ) (fig $\mathrm{l}$ ).

This 55\% increase in cognitive processing speed in the Parkinson's disease group across sessions was highly significant ( set size $\times$ run interaction: $F(1,9)=25.4, p<0.0001)$ and occurred in all 10 patients (fig 2 ). In contrast, the control subjects showed no significant change in slope across sessions ( set size $\times$ run interaction: $F(1,9)=0.002, p=0.97$; session 1 , $\mu=0.061$ (0.015); session 2, $\mu=0.061$ (0.015)) (figs 1 and 2). Nor did the intercept of the control group change across sessions $(\mathrm{F}(\mathrm{l}, 9)=0.012 ; \mathrm{p}=0.91 ;$ session $\mathrm{l}, \mu=0.544$ (0.052); session $2, \mu=0.541(0.051))$. The groups did not differ in the intercept-the motor component of the response time-at either session (group effect, $\mathrm{F}(1,18)=0.57, \mathrm{p}=0.45$ at the first session; $F(1,18)=2.1, p=0.16$ at second session).

On a post-hoc basis, we examined whether the improvement in the working memory component (slope) of the Parkinson's disease group occurred during the first session or between the two sessions. Comparing the performance of the patients between the first and second blocks of the first session, there was no significant improvement (block $\times$ set size interaction: $F(1,9)=2.0, p=0.15)$. However, there was a significant change between the second block of the first session and the first block of the second session (block $\times$ set size interaction: $F(1,9)=15.6, p<0.0001$ ) (fig 3). This indicates that the improvement in working memory occurred between sessions. Control subjects showed no significant changes either within or between test sessions $(p>0.24$ for all comparisons of individual test blocks).

\section{Accuracy}

There was no effect of dopaminergic repletion on accuracy in the Parkinson's disease group $(F(1,9)=0.18, p=0.68)$. Collapsed across sessions and working memory load, there
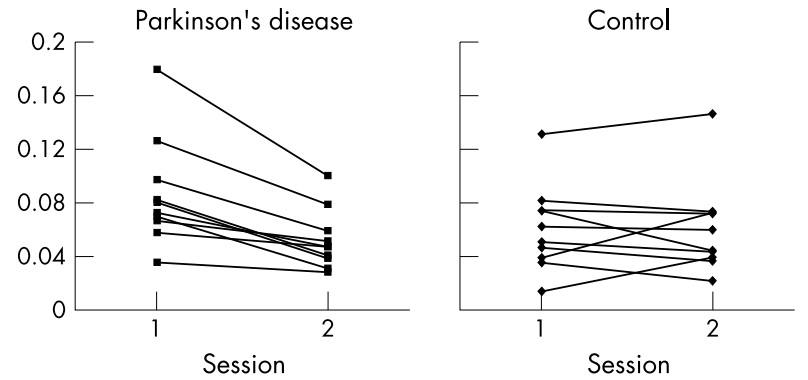

Figure 2 The slope (ms/number of items) or cognitive component of the response time for each subject at each session. Parkinson's disease patients are on the left and control subjects on the right. All 10 subjects with Parkinson's disease showed a decrease in slope (more efficient cognitive component) in session 2. There was no consistent change in the control subjects.

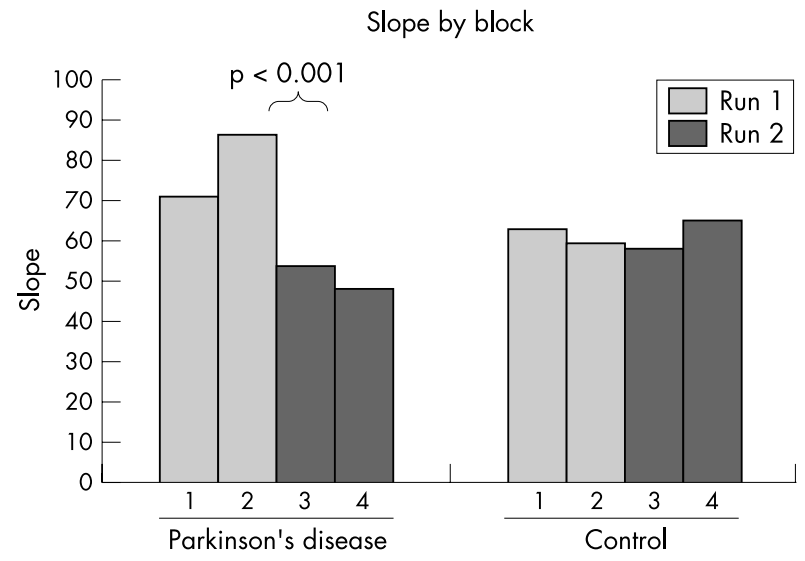

Figure 3 The slope (working memory component of the response time) for each block is shown. Blocks 1 and 2 (hatched bars) make up the first run; blocks 3 and 4 (solid bars) make up the second run, performed one week later. Parkinson's disease patients had a lower slope between blocks 2 and 3 -that is, between runs $(p<0.001$, $p>0.1$ for all other comparisons).

was no group difference in accuracy $(\mathrm{F}(1,18)=1.7, \mathrm{p}=0.20)$; control, $\mu=96.45$ (2.37); Parkinson's disease, $\mu=94.17$ (5.07). However, there was an interaction of group and set size (working memory load) $(\mathrm{F}(2,36)=3.31, \mathrm{p}=0.048)$. Patients with Parkinson's disease made more errors than controls only at the highest working memory load (set size of five digits) $(\mathrm{t}(38)=2.29, \mathrm{p}=0.03)$. As with response time, subjects with Parkinson's disease were less accurate than controls in the high working memory load during the first session $(\mathrm{t}(18)=2.17, \mathrm{p}=0.044)$, but performed as accurately as controls during the second session $(\mathrm{t}(18)=0.94, \mathrm{p}=0.349)$. This improvement in performance in the Parkinson's disease group from the first to the second session approached significance $(t(9)=1.95, p=0.084)$, while the performance of the controls did not change $(\mathrm{t}(9)=0.0, \mathrm{p}=1.0)$. These data show that the Parkinson's disease group had improved working memory in terms of speed at the second session and a trend toward improved performance in terms of accuracy, ruling out a speed-accuracy trade off.

\section{DISCUSSION}

The findings show that the slowed response time of patients with Parkinson's disease relative to controls during working memory performance is a function of a cognitive slowing and not of motor slowing. Contrary to our initial hypothesis, this cognitive slowing was not affected by dopaminergic state; rather, it resolved over time. Moreover, the pattern of improvement between, rather than within, sessions suggests that 
patients with Parkinson's disease have delay in "proceduralising" their working memory performance.

Cognitive deficits in Parkinson's disease are sometimes attributed to confounding variables such as comorbid depression or incipient dementia. In this study, subjects with these conditions were excluded on the basis of stringent criteria after screening with detailed mental status and depression rating tests. We were also able to eliminate the potential confounding of motor slowing in assessing cognitive performance by using the SIRP. The SIRP dissociates motor and cognitive aspects of performance. Finally, we used a fixed set form of the SIRP, which emphasises working memory maintenance and minimises manipulative and set switching requirements.

We found that the motor component of the response time did not differ between the Parkinson's disease group and the controls and did not change with dopaminergic repletion. The findings with respect to dopaminergic repletion are not unprecedented. Some studies have found no change in simple response time (as opposed to choice response time or movement time) with dopaminergic repletion in patients with Parkinson's disease ${ }^{33}{ }^{34}$ while others have found a decrease in simple response time. ${ }^{35}{ }^{36}$ Studies comparing subjects with Parkinson's disease with controls have generally found a longer simple response time in the "off" state in the former (reviewed by Jahanshahi et al ${ }^{37}$ ), but there are exceptions. Using the SIRP, Smith and colleagues did not find slowing of the motor component of the response time in a group of dopaminergically depleted patients with Parkinson's disease. ${ }^{24}$ Specific task characteristics, including the type of motor response required (for example button press, as in the current study, versus a response that requires a larger movement) may provide a partial explanation of these contrasting findings. ${ }^{38}$ In addition, although the motor component of the SIRP is conceptually similar to simple response time, it differs in that it is a derived measure (the $y$ intercept of the regression line) that isolates the motor component of response time.

Another possible reason for contrasting findings concerns the severity of the dopaminergic deficit. The subjects with Parkinson's disease in the current study were mildly affected. The long duration response to levodopa is more prominent in the earlier stages of Parkinson's disease and can last for several days. $^{39}$ Although the overnight 12 hour drug withdrawal in this study was sufficient to cause a significant increase in motor impairment (UPDRS), it may not have been sufficient to affect the motor component of response time, which did not differ from the control subjects.

We also found that dopaminergic state did not alter cognitive slowing. Again, it is possible that this finding is related to the state of dopaminergic deficiency, as discussed above. A dopamine effect on working memory might also have been masked by the improvement seen across sessions. If this were the case, the dopamine effect would have to have been considerably smaller than the session effect.

Reports on the effects of dopaminergic repletion on working memory performance in Parkinson's disease are inconsistent. Studies report improvement, ${ }^{20} 4041$ no effect, ${ }^{7842}$ and worse performance..$^{22}$ Moderate levels of dopamine in the DLPFC are hypothesised to improve cortical efficiency (the degree of neuronal activity required to perform a task), while excessive levels may impair efficiency. ${ }^{42}$ The finding that dopamine levels are actually increased in early untreated Parkinson's disease ${ }^{16}$ suggests that dopaminergic repletion in such cases may lead to excessive levels in the DLPFC. Differences in DLPFC dopamine levels may give rise to heterogeneous effects of dopamine repletion on working memory. Working memory task requirements may also influence study outcome. Working memory involves both maintenance and manipulation. Dorsal prefrontal cortex regions are thought to be preferentially recruited for manipulation and ventral regions for maintenance. ${ }^{43} 44$ Tasks emphasising maintenance, such as the SIRP, may be less affected by dopaminergic repletion owing to their greater dependence on more ventral prefrontal cortex regions that are less affected in early Parkinson's disease.

Although there was no change in task performance with dopaminergic repletion, the subjects with Parkinson's disease showed a significant $55 \%$ improvement in the cognitive component of the response time across sessions. We hypothesise that this improvement reflects a delay in proceduralising the cognitive aspects of task performance. Procedural learning refers to the acquisition of knowledge expressed through experience induced changes in performance. ${ }^{45}$ The accurate performance of the patients with Parkinson's disease confirmed that they do not have difficulty comprehending the task; rather, they may have deficits in forming the programs to automate and optimally execute the task. Control subjects quickly form stable and efficient programs to perform this task, as reflected in their stable performance both within and between sessions. In contrast, subjects with Parkinson's disease appear to have a delay in optimising these programs. Several studies have shown that patients with Parkinson's disease have deficits in procedural learning tasks. ${ }^{46-48}$ Our findings suggest that these procedural learning deficits can impair their performance of tasks that primarily tap other cognitive realms. However, with time and practice, affected individuals appear to be able to achieve normal performance levels.

An intriguing finding is that the improvement in the individuals with Parkinson's disease occurred during the intervening week between the sessions rather than within either session. While it is possible that with additional continuous exposure during the first session their performance would have improved, certain tasks require an intervening period of time and sleep for optimal performance. For instance, visual discrimination learning shows maximal improvement 48 to 96 hours after initial training, even without intervening practice. ${ }^{49}$ Stickgold and colleagues recently confirmed that sleep after training is required for this improvement. ${ }^{50}$ Performance of a different procedural learning task-the serial reaction time task-alters regional brain activity during the period of sleep that follows exposure. A subset of the brain regions active during task performance appears to come back on line during REM sleep. ${ }^{51}$ Similarly, Walker et al have recently shown sleep dependent inprovement on a motor finger tapping task. ${ }^{52}$ The role of extended exposure to the SIRP and the time course of the improvement is the basis of an ongoing study in our laboratory.

Our findings suggest that procedural learning deficits may explain impairments in working memory performance in Parkinson's disease. As this finding was unexpected, it requires replication. The pattern of neuropathology in Parkinson's disease could be seen as consistent with this hypothesis. Procedural learning is thought to be subserved by a frontostriatal network parallel to the working memory circuit, with cortical nodes in the primary motor, premotor, and supplementary motor cortices, and the striatal node in the putamen. ${ }^{13554}$ Working memory circuits and procedural learning circuits have separate striatal nodes, the caudate being preferentially involved in working memory. ${ }^{55}$ Impaired dopaminergic projections from the substantia nigra to the caudate and putamen (the nigrostriatal pathway) form the pathophysiological basis of Parkinson's disease. Dopaminergic deficiencies are significantly more severe in the putamen than in the caudate. ${ }^{56}$ As the putamen acts as the striatal node in the procedural learning circuit, patients with Parkinson's disease may have more difficulty with the procedural component of cognitive tasks.

If confirmed, these results have important implications for studies of cognitive function in Parkinson's disease. The delayed procedural learning found in our study could underlie impaired performance in other cognitive tasks. Cognitive deficits in 
patients with Parkinson's disease found in other studies may resolve with practice, over time. The relation between procedural and working memory components of task performance remains to be elucidated and may be critical in determining the basis of cognitive impairments in Parkinson's disease.

\section{ACKNOWLEDGEMENTS}

We would like to thank Michael Alexander MD and Alvaro Pascual-Leone MD, PhD for their thoughtful comments on the manuscript. This work was supported by the Goldberg Parkinson Project (DZP) and NIH grants NS10980 (DZP) and K23MH01829 (DSM).

\section{Authors' affiliations}

D Z Press, D J Mechanic, D Tarsy, Behavioral Neurology Unit and Movement Disorders Center, Department of Neurology, Beth Israel Deaconess Medical Center, Boston, Massachusetts, USA D S Manoach, Department of Psychiatry, Massachusetts General Hospital-East and Athinoula A Martinos Center for Biomedical Imaging, Harvard Medical School, Boston

Competing interests: none declared.

\section{REFERENCES}

1 Aarsland D, Andersen K, Larsen JP, et al. Risk of dementia in Parkinson's disease: a community-based, prospective study. Neurology 2001:56:730-6.

2 Kuzis G, Sabe L, Tiberti C, et al. Cognitive functions in major depression and Parkinson disease. Arch Neurol 1997;54:982-6.

3 Cooper JA, Sagar HJ, Doherty SM, et al. Different effects of dopaminergic and anticholinergic therapies on cognitive and motor function in Parkinson's disease. A follow-up study of untreated patients. Brain 1992;115:1701-25.

4 Cooper JA, Sagar HJ, Jordan N, et al. Cognitive impairment in early, untreated Parkinson's disease and its relationship to motor disability. Brain 1991;114:2095-122.

5 Levin BE, Llabre MM, Weiner WJ. Cognitive impairments associated with early Parkinson's disease. Neurology 1989;39:557-61

6 Taylor AE, Saint-Cyr JA, Lang AE. Frontal lobe dysfunction in Parkinson's disease. The cortical focus of neostriatal outflow. Brain 1986; 109:845-83.

7 Cools R, Barker RA, Sahakian BJ, et al. Mechanisms of cognitive se flexibility in Parkinson's disease. Brain 2001;124:2503-12.

8 Gotham A, Brown R, Marsden C. "Frontal" cognitive function in patients with Parkinson's disease "on" and "off" levodopa. Brain 1988;111:299-321.

9 Morris RG, Downes JJ, Sahakian BJ, et al. Planning and spatial working memory in Parkinson's disease. J Neurol Neurosurg Psychiatry 1988;51:757-66.

10 Owen AM, Iddon JL, Hodges JR, et al. Spatial and non-spatial working memory at different stages of Parkinson's disease. Neuropsychologia 1997;35:519-32.

11 Postle BR, Jonides J, Smith EE, et al. Spatial, but not object, delayed response is impaired in early Parkinson's disease. Neuropsychology 1997:11:171-9.

12 Bradley VA, Welch JL, Dick DJ. Visuospatial working memory in Parkinson's disease. J Neurol Neurosurg Psychiatry 1989;52:1228-35.

13 Alexander GE, DeLong MR, Strick PL. Parallel organization of functionally segregated circuits linking basal ganglia and cortex. Annu Rev Neurosci 1986:9:357-81.

14 Williams GV, Goldman-Rakic PS. Modulation of memory fields by dopamine D1 receptors in prefrontal cortex. Nature 1995;376:572-5.

15 Collins P, Wilkinson LS, Everitt BJ, et al. The effect of dopamine depletion from the caudate nucleus of the common marmoset (Callithrix jacchus) on tests of prefrontal cognitive function. Behav Neurosci 2000;114:3-17

16 Kaasinen V, Nurmi E, Bruck A, et al. Increased frontal [[(18)F]]fluorodopa uptake in early Parkinson's disease: sex differences in the prefrontal cortex. Brain 2001;124:1125-30.

17 Whitehouse PJ, Hedreen JC, White $\mathrm{CL}$, et al. Basal forebrain neurons in the dementia of Parkinson disease. Ann Neurol 1983;13:243-8.

18 Zweig RM, Cardillo JE, Cohen M, et al. The locus ceruleus and dementia in Parkinson's disease. Neurology 1993;43:986-91.

19 Pillon B, Dubois B, Cusimano G, et al. Does cognitive impairment in Parkinson's disease result from non-dopaminergic lesions? J Neurol Neurosurg Psychiatry 1989;52:201-6.

20 Lange KW, Robbins TW, Marsden CD, et al. L-dopa withdrawal in Parkinson's disease selectively impairs cognitive performance in tests sensitive to frontal lobe dysfunction. Psychopharmacology 1992; 107:394-404.

21 Sternberg S. High-speed scanning in human memory. Science 1966;153:652-4.

22 Poewe W, Berger W, Benke T, et al. High-speed memory scanning in Parkinson's disease: adverse effects of levodopa. Ann Neurol 1991;29:670-3.
23 Rafal RD, Posner MI, Walker JA, et al. Cognition and the basal ganglia: separating mental and motor components of performance in Parkinson's disease. Brain 1984;107:1083-94.

24 Smith MC, Goldman WP, Janer KW, et al. Cognitive speed in nondemented Parkinson's disease. J Int Neuropsychol Soc 1998:4:584-92

25 Manoach DS, Schlaug G, Siewert B, et al. Prefrontal cortex fMRI signal changes are correlated with working memory load. Neuroreport 1997;8:545-9

26 Rypma B, Prabhakaran V, Desmond JE, et al. Load-dependent roles of frontal brain regions in the maintenance of working memory. Neuroimage 1999;9:216-26.

27 Kristofferson MW. Effects of practice on character-classification performance. Can J Psychol 1972;26:54-60.

28 Gelb DJ, Oliver E, Gilman S. Diagnostic criteria for Parkinson disease. Arch Neurol 1999:56:33-9.

29 Hoehn MM, Yahr MD. Parkinsonism: onset, progression and mortality Neurology 1967; 17:427-42.

30 Beck A, Rial W, Rickels K. Short Form of Depression Inventory: cross-validation. Psychol Rep 1974;34:1184-6.

31 Coblentz J, Mattis S, Zingesser L, et al. Presenile dementia. Clinical aspects and evaluation of CSF dynamics. Arch Neurol 1973;29:299-308.

32 Blair JR, Spreen O. Predicting premorbid IQ: a revision of the National Adult Reading Test. Clin Neuropsychol 1989;3:129-36.

33 Pullman SL, Watts RL, Juncos JL, et al. Dopaminergic effects on simple and choice reaction time performance in Parkinson's disease. Neurology 1988:38:249-54

34 Pullman SL, Watts RL, Juncos JL, et al. Movement amplitude choice reaction time performance in Parkinson's disease may be independent of dopaminergic status. I Neurol Neurosurg Psychiatry 1990:53:279-83.

35 Harrison J, Henderson L, Kennard C. Abnormal refractoriness in patients with Parkinson's disease after brief withdrawal of levodopa treatment. J Neurol Neurosurg Psychiatry 1995;59:499-506.

36 Montgomery EB, Nuessen J. The movement speed/accuracy operator in Parkinson's disease. Neurology 1990:40:269-72

37 Jahanshahi M, Brown RG, Marsden CD. Simple and choice reaction time and the use of advance information for motor preparation in Parkinson's disease. Brain 1992;115:539-64.

38 Gauntlett-Gilbert J, Brown VJ. Reaction time deficits and Parkinson's disease. Neurosci Biobehav Rev 1998;22:865-81.

39 Hauser RA, Koller WC, Hubble JP, et al. Time course of loss of clinica benefit following withdrawal of levodopa/carbidopa and bromocriptine in early Parkinson's disease. Mov Disord 2000;15:485-9.

40 Fournet N, Moreaud O, Roulin JL, et al. Working memory functioning in medicated Parkinson's disease patients and the effect of withdrawal of dopaminergic medication. Neuropsychology 2000;14:247-53.

41 Kulisevsky J, Avila A, Barbanoj M, et al. Acute effects of levodopa on neuropsychological performance in stable and fluctuating Parkinson's disease patients at different levodopa plasma levels. Brain 1996;119:2121-32.

42 Mattay VS, Tessitore A, Callicott JH, et al. Dopaminergic modulation of cortical function in patients with Parkinson's disease. Ann Neurol 2002;51:156-64

43 Petrides $\mathbf{M}$. Functional organization of the human frontal cortex for mnemonic processing. Evidence from neuroimaging studies. Ann NY Acad Sci 1995:769:85-96.

44 D'Esposito M, Postle BR, Ballard D, et al. Maintenance versus manipulation of information held in working memory: an event-related fMRI study. Brain Cogn 1999;41:66-86.

45 Gabrieli JD. Cognitive neuroscience of human memory. Annu Rev Psychol 1998;49:87-115.

46 Haaland KY, Harrington DL, O'Brien S, et al. Cognitive-motor learning in Parkinson's disease. Neuropsychology 1997;1 1:180-6.

47 Pascual-Leone A, Grafman J, Clark K, et al. Procedural learning in Parkinson's disease and cerebellar degeneration. Ann Neurol 1993:34:594-602.

48 Sommer M, Grafman J, Clark K, et al. Learning in Parkinson's disease: eyeblink conditioning, declarative learning, and procedural learning. Neurol Neurosurg Psychiatry 1999;67:27-34.

49 Stickgold R, Whidbee D, Schirmer B, et al. Visual discrimination task improvement: a multi-step process occurring during sleep. J Cogn Neurosci 2000;1 2:246-54

50 Stickgold R, James L, Hobson JA. Visual discrimination learning requires sleep after training. Nat Neurosci 2000;3:1237-8.

51 Maquet $\mathbf{P}$, Laureys $\mathrm{S}$, Peigneux $\mathrm{P}$, et al. Experience-dependent changes in cerebral activation during human REM sleep. Nat Neurosci 2000;3:831-6

52 Walker MP, Brakefield T, Morgan A, et al. Practice with sleep makes perfect: sleep dependent motor skill learning. Neuron 2002;35:205-11.

53 Hoover JE, Strick PL. Multiple output channels in the basal ganglia. Science 1993;259:819-21.

54 Hoover JE, Strick PL. The organization of cerebellar and basal ganglia outputs to primary motor cortex as revealed by retrograde transneurona transport of herpes simplex virus type 1. J Neurosci 1999;19:1446-63.

55 Rinne JO, Portin R, Ruottinen $\mathrm{H}$, et al. Cognitive impairment and the brain dopaminergic system in Parkinson disease: [[18F]]fluorodopa positron emission tomographic study. Arch Neurol 2000;57:470-5

56 Brooks DJ, Ibanez V, Sawle GV, et al. Differing patterns of striatal $18 \mathrm{~F}$-dopa uptake in Parkinson's disease, multiple system atrophy, and progressive supranuclear palsy [[see comments]]. Ann Neurol 1990;28:547-55. 